\title{
Contribuições de Fisher para a compreensão do desenvolvimento da percepção corporal
}

\author{
Patríaa RussoLiteRibeiro- UnivesidadeEstadual deCaminas, Caminas SãoPaulo, Brasil \\ Maria da Consdação Gomes Cunha Femandes Tavares- UnivesidadeEstadual deCaminas, Campinas, SãoPaulo, Brasil \\ Aletha Silva Cadano- UnivesidadeEstadual deCampinas, Campinas, SãoPaulo, Brasil
}

\begin{abstract}
$\overline{\text { Resumo }}$
O objetivo deste artigo é apresentar as ideias de Fisher sobre o desenvolvimento da percepção corporal, através da abordagem da imagem corporal. Foram consultados três livros publicados por Fisher, que não são traduzidos para a língua portuguesa. Fisher propôs que a habilidade de perceber o espaço do próprio corpo se inicia na infância, por meio das primeiras experiências corporais, evoluindo para avaliações cada vez mais complexas e processos de diferenciação eumundo. 0 conceito de fronteiras corporais proposto pelo autor complementa essas ideias. Os padrões de percepção corporal servem para específicos propósitos de defesa, pois vão informar se determinado estímulo deve ser evitado ou não. A abordagem da imagem corporal permite aos profissionais da educação e saúde atuarem de forma mais individualizada e humana.
\end{abstract}

Palavasdhaves Percepção corporal; Imagem corporal; D esenvolvimento psicomotor.

The development of the body perception, according to Fisher

\begin{abstract}
The aim of this paper is present Fisher's ideas about the development of the body perception, by addressing body image. Three of the books written by Fisher, which were not translated to Portuguese, were used. Fisher proposed that the ability to percept the space of the own body begins in childhood, through the first body experiences, developing to more complex evaluations of the physical proprieties and self-world differentiation processes. The concept of bodily boundaries proposed by the author complements these ideas. The patterns of body perception serve specific purposes of defense, as they will inform whether a stimulus should be avoided or not. The body image approach allows health and education professionals to act in a more individualized and human way.
\end{abstract}

Keguards Body perception; Body image; Psychomotor development.

\section{Contribuciones de Fisher para la comprensión del desarrollo de la percepción corporal}

\begin{abstract}
Resumen
El objetivo de esta investigación es presentar las ideas de Fisher respecto al desarrollo de la percepción corporal, a través del abordaje de la imagen corporal. Fueron consultados tres libros publicados por Fisher, que no son traducidos para la lengua portuguesa. Fisher propuso que la habilidad de percibir el espacio del propio cuerpo se inicia en la infancia, por medio de las primeras experiencias corporales, evolucionando para evaluaciones cada vez más complejas y procesos de diferenciación YO-mundo. El concepto de fronteras corporales propuesto por el autor complementa esas ideas. Los patrones de percepción corporal sirven para específicos propósitos de defensa, puesto que van a informar si determinado estímulo debe ser evitado o no. El abordaje de la imagen corporal permite a los profesionales de la educación y salud actuaren de forma más individualizada y humana.

Palabras dave Percepción corporal; Imagen corporal; D esarrollo psicomotor.
\end{abstract}

0 termo imaggm caparal refere-se à representação mental da identidade corporal do indivíduo. É um fenômeno complexo, multidimensional e dinâmico, que engloba aspectos fisiológicos, psicológicos e sociais inerentes a toda e qualquer experiência corporal vivenciada pelo indivíduo (Schilder, 1999). Os primeiros pesquisadores da imagem corporal foram, então, os neurologistas, com destaque para Henry Head e Paul Schilder, que começaram a investigar 0 tema em meados do século XX.

A percepção corporal começou a ser pesquisada enquanto uma dimensão da imagem corporal no século XX. Os neurologistas dessa época estavam interessados em compreender fenômenos como o membro fantasma e a anosognosia, considerados maneiras distorcidas de percepção do próprio corpo. A ideia da existência de uma imagem corporal proporcionava aos pesquisadores um melhor entendimento para esses casos clínicos. Partiram, então, do pressuposto de que existe um esquema central da imagem corporal capaz de registrar as percepções corporais e integrálas, e as distorções dessas percepções estariam relacionadas a danos cerebrais específicos (Fisher, 1990).

Seymour Fisher foi um dos primeiros psicólogos a investigar o fenômeno da imagem corporal de maneira aprofundada. Durante mais de trinta anos, dedicou-se intensamente aos estudos em imagem corporal sob uma perspectiva psicodinâmica e incluiu em seu trabalho investigações acerca da percepção corporal, compreendida como uma das dimensões da imagem corporal. Uma das principais preocupações de Fisher era com a dispersão e a desarticulação entre as pesquisas sobre imagem corporal que estavam sendo produzidas durante a segunda metade do século XX. Por isso, o autor se empenhou em agrupá-las, fazer 0 cruzamento dos dados, analisá-los e organizar os conhecimentos ali emergidos para difundi-los no meio científico. Como consequência desse trabalho, publicou 
trës livros de revisäo critica da literatura, onde apresenta, de forma sistematizada, inúmeros achados dos pesquisadores da área, bem como dados resultantes de pesquisas que realizou (Fisher, 1970, 1986; Fisher \& Cleveland, 1968). Por ser considerado peça-chave no desenvolvimento das pesquisas em imagem corporal (Cash, 2004; Cash \& Pruzinsky, 2002) e por ter sistematizado os conhecimentos produzidos sobre percepção corporal especificamente, é fundamental, pertinente e necessário ampliar a difusão dos conhecimentos de Fisher. Portanto, o objetivo do presente trabalho é apresentar os aspectos do desenvolvimento da percepção corporal propostos por Fisher aos pesquisadores e profissionais da área no Brasil, uma vez que nenhum de seus livros que tratam do fenômeno da imagem corporal e suas dimensões foi traduzido para a língua portuguesa.

Como metodologia de trabalho, foi realizada uma pesquisa do tipo bibliográfica, baseada nos rigores científicos propostos por Salvador (1982), Cervo e Bervian (1975) e Lakatos e Macconi (1991). As etapas da pesquisa envolveram coleta, análise e interpretação de dados, realizados mediante levantamento bibliográfico e leituras informativa e interpretativa, respectivamente.

O primeiro livro publicado pelo autor foi Body imageand pasonality (1968/ 1958), escrito com Sidney E. Cleveland, no qual propõem que o corpo é um objeto psicológico, que o indivíduo organiza suas percepções corporais e que estas afetam seu comportamento. A publicação do livro obteve grande impacto no meio científico e propiciou o reconhecimento da experiência corporal como objeto de pesquisa da psicologia moderna.

O livro Booty experience in fantasy and bethevior (1970) foi direcionado à temática da organização das experiências associadas à percepção e aos significados que o indivíduo atribui ao seu próprio corpo, e do reconhecimento de que os valores e as expectativas que o indivíduo possui interferem diretamente no seu comportamento.

Em 1986, Fisher lançou os dois volumes do Dedepment and structure of thebooyimage 0 volume 1 do livro contém a revisão detalhada e a análise da literatura sobre imagem corporal publicada entre os anos de 1969 a 1985. 0 volume 2 contém uma descrição geral do extenso trabalho realizado para avaliar os principais conceitos teóricos que Fisher propôs a respeito do funcionamento da imagem corporal. Ele os separa em quatro categorias: organização das fronteiras da imagem corporal, atribuição de significados a partes específicas do corpo, consciência geral do corpo e distorções na percepção corporal.
A partir do estudo desses trës livros, toi possível sistematizar aspectos da percepção corporal reconhecidos por Fisher como fundamentais para 0 desenvolvimento de todo indivíduo.

\section{0 desenvolvimento da percepção corporal - os conhecimentos sobre o corpo}

0 referencial teórico assumido por Fisher teve como base as proposições de Schilder (1999), que concebia o conceito de corpo formulado a partir da ideia de uma experiência psicológica, focada nas atitudes e sentimentos que cada indivíduo possui sobre seu próprio corpo. Este fenômeno, denominado imagem corporal, refere-se às experiências individuais subjetivas do corpo e à maneira como o indivíduo as organiza ao longo de seu desenvolvimento. Para Schilder, a maneira pela qual o indivíduo lida com esta difícil tarefa de organizar suas sensações corporais vem a ser a primeira forma de organização de padrões de interpretação do mundo que o cerca. As percepções corporais, assim, foram entendidas como as primeiras experiências de corpo vividas pela criança. Dessa forma, seriam fundamentais para a construção da sua imagem corporal.

Para Fisher (1986), Schilder foi, provavelmente, 0 teórico da personalidade que atribuiu maior importância à imagem corporal no processo de desenvolvimento do ser humano. Segundo Schilder (1999), a criança nasce sem a consciência de seu corpo, sem diferenciar esse corpo dos demais objetos. O mundo e 0 corpo são experiências indissociáveis, ou seja, uma experiência não é possível sem a outra. Foi além das perspectivas freudianas, considerando que outras áreas do corpo, como os olhos e a pele, também são pontos de referência proeminentes, assim como as zonas erógenas, na construção das imagens corporais da criança. Para esse autor, um desejo específico está associado a uma zona específica do corpo e nesse momento, essa zona torna-se 0 centro da sua imagem corporal. Considerou que a dor e o controle motor sobre os membros são fatores fundamentais para a criação da imagem corporal e para sua condição dinâmica de estar constantemente sendo destruída e reconstruída.

Fisher (1986) aprofundou suas investigações a respeito do desenvolvimento das experiências corporais. Diferentes estudos (Gellert, 1960; Piaget, 1960; Williams, 1979) indicavam haver diferenças marcantes entre a percepção corporal da criança e do adulto, e Fisher buscou pormenorizar como se dá este processo de desenvolvimento, por meio da análise dos dados obtidos em tais pesquisas.

Segundo Piaget (1954), a criança começa a construir uma noção básica do mundo através das suas 
experiências sensório-motoras. O corpo em ação, movimentando-se no espaço em busca de um objeto, oferece um padrão para a estruturação do mundo. Assim, as primeiras experiências são de caráter corporal e se organizam a partir da espacialidade do corpo. Piaget considera que a ação do corpo é a matéria-prima para todas as adaptações intelectuais e perceptivas. Nesse sentido, ao longo do seu desenvolvimento, a criança internaliza tais ações. A internalização envolve a criação de imagens que representam as sequências sensório-motoras.

A teoria de Piaget sugere que antes de a criança aprender a imitar um comportamento, ela precisa ter certo conhecimento da topografia do seu corpo, tratálo como um objeto, aprender suas qualidades e propriedades de locomoção no espaço. Apesar de haver algumas conotações ao fenômeno da imagem corporal no que se refere a como a criança desenvolve o conceito de espaço e suas coordenadas em geral, tendo o corpo como referência central, muitas das observações de Piaget sobre o comportamento infantil estão claramente relacionadas ao conceito de esquema corporal (Fisher, 1986).

Fisher apontou Werner (1957) como pioneiro em atribuir importância ao corpo enquanto um construto psicológico. Werner sugeriu que as experiências corporais infantis são fundamentais para a construção do seu mundo e colorem as suas percepções desse mundo. Ele descreve como a percepção corporal oferece estrutura para a definição do el e como a aquisição da linguagem e as habilidades cognitivas estão fortemente relacionadas à imagem corporal e às sensações corporais.

Freud (1908) também considerou a importância das atitudes corporais em sua teoria. Para ele, as experiências corporais são a base para a formação do ego. Em sua teoria da libido, ele correlaciona 0 desenvolvimento da personalidade a um processo que envolve a transferência sucessiva de atenção e energia investidas de uma parte para outra do corpo (fases oral, anal, genital). Assim, Fisher (1986) considera que suas formulações foram importantes para o campo da imagem corporal quando abordou questões como a criança aprender a distinguir eventos internos do seu corpo dos eventos externos, as sensações desencadeadas em áreas específicas do corpo pelas experiências de socialização, a ansiedade causada por possíveis danos corporais e relacionada às ameaças parentais, a confusão sobre a estrutura corporal causada pela descoberta das diferenças sexuais, entre outras. As formulações freudianas sobre 0 desenvolvimento da criança estavam impregnadas de referências corporais.
Erikson (1950) aproximou-se de Freud quando formulou um elaborado esquema sobre 0 desenvolvimento humano, no qual cada atitude fundamental do sujeito está associada a atitudes perante um sistema corporal específico. Este "encontro" com a zona corporal estabelece os eventos precedentes e as expectativas de vida do sujeito, e isso constitui o seu desenvolvimento.

Fisher (1986) consultou ainda outros teóricos, como Witkin, Dyk, Faterson, Goodenough e Karp (1962), Kohlberg (1966), Reich (1949), Klein (1932), que propuseram ideias interessantes sobre 0 desenvolvimento da imagem corporal, a partir das quais foi possível avançar na compreensão desse fenômeno. Muitos desses autores assumiram que a criança inicia seu desenvolvimento com nenhuma consciência de que seu corpo é uma entidade separada do mundo exterior; que o seu conceito de corpo tornase cada vez mais diferenciado e complexo ao longo dos anos e que essa diferenciação inicia a partir da noção de possuir um contorno ou borda corporal; que o corpo é a referência principal da criança na aprendizagem da espacialidade; que a formação da imagem corporal se dá ao longo de uma série de estágios nos quais, em cada um deles, existe o foco em uma determinada parte do corpo (ex.: boca, ânus, genitais); que a descoberta da diferença sexual é uma etapa fundamental na estruturação do conceito de corpo; que as atitudes corporais da criança são bastante influenciadas pelos valores parentais; que em certas fases do desenvolvimento, a imagem corporal experiencia instabilidade e que quando a criança se torna adolescente em direção à fase adulta, os fatores cognitivos tornam-se mais influentes do que a experiência corporal nas tomadas de decisão.

Fisher correlacionou essas formulações ao conceito proposto por ele denominado de fronteiras coparais (Fisher \& Cleveland, 1968). Fronteiras corporais referem-se às diferentes maneiras com que as pessoas atribuem "firmeza" e "definição" à sua superfície corporal. Esse termo propõe a ideia de que as bordas do corpo são responsáveis por intermediar as relações do indivíduo com 0 mundo. Assim, as fronteiras corporais representam aquilo que 0 indivíduo permite ou não permear por sua imagem corporal. Essas fronteiras oferecem a ele a noção de um corpo seguro, protegido e menos vulnerável às ameaças que colocam em risco a integridade de seu corpo.

Partindo do pressuposto de que 0 desenvolvimento da percepção corporal é um aspecto primordial para a construção da imagem corporal, Fisher foi buscar nos achados de diferentes autores as respostas para compreender: (a) quando é que a criança 
torna-se consciente da exıstencia de um espaço corporal único que representa o seu aue como se dá 0 processo de diferenciação das partes internas e externas de seu corpo e de seu próprio corpo em relação aos demais objetos; (b) a aprendizagem da espacialidade e da direcionalidade do corpo; (c) 0 potencial da atratividade corporal em afetar as respostas dos outros, com os quais a criança está interagindo; (d) 0 desenvolvimento da organização das percepções corporais; (e) a relação entre a imagem corporal e a aquisição das habilidades cognitivas; (f) o papel da experiência corporal no desenvolvimento do autoconceito da criança; (g) o papel do objeto transicional de fornecer sensação de segurança e alívio quando colocado perto do corpo; (h) as mudanças na percepçao corporal, no corpo e nas atitudes do adolescente; (i) a primeira menstruação e (j) a puberdade.

As pesquisas analisadas por Fisher trazem 0 interesse dos autores em explorar as mudanças no desenvolvimento da percepção corporal. Segundo o autor, os métodos utilizados foram diversos e houve certa dificuldade para organizar os resultados. Mesmo assim, conseguiu delinear um esquema de dados que emergiram de suas análises. A Tabela 1 sintetiza os aspectos peculiares das diferentes fases do desenvolvimento da criança que o autor apresentou no primeiro volume do seu livro Dedepment and structure of theboodyimage(Fisher, 1986).

Tabela 1. O desenvolvimento da percepção corporal nas diferentes fases da criança

\begin{tabular}{|c|c|}
\hline Anos & D esenvolvimento \\
\hline Até 3 a. & $\begin{array}{l}\text { - imagem no espelho: única representação de si, } \\
\text { - identifica: partes do corpo, espaço, padrões de atratividade corporal, } \\
\text { - consciência da identidade sexual e do próprio espaço corporal, } \\
\text { - 1ano: objetos transicionais; 3anos: fantasias com ameaças corporais. }\end{array}$ \\
\hline 4-5 a. & $\begin{array}{l}\text { - consciência: esquema para memorizaçáo de aspectos relevantes da organização corporal; 5anos: } \\
\text { esquema corporal central: estratégias corporais para respostas, } \\
\text { - melhora na habilidade de reconhecer e representar formas humanas e/ ou tridimensionais, maior } \\
\text { conhecimento das partes do corpo, } \\
\text { - diferenciação da imagem corporal: habilidade de avaliar preferências e interesses pessoais, atitudes } \\
\text { corporais diferenciadas. }\end{array}$ \\
\hline 6-8 a. & $\begin{array}{l}\text { - distinção direita-esquerda e bom julgamento do tamanho das partes do corpo, } \\
\text { - compreende: diferenças entre os genitais, importância do coração, } \\
\text { - início da estruturação das fronteiras corporais: "corpo seguro". }\end{array}$ \\
\hline 9-10 a. & $\begin{array}{l}\text { - 9anos: novo pico de aceleração na construção da imagem corporal, } \\
\text { - todas as habilidades sensório-motoras continuam a melhorar, } \\
\text { - 10anos: imagem corporal estável. }\end{array}$ \\
\hline $11-12 \mathrm{a}$. & $\begin{array}{l}\text { - total consciência da localização e função da maioria das partes do corpo, } \\
\text { - garotas: menstruação: aumento de sentimentos de feminilidade. }\end{array}$ \\
\hline $\begin{array}{l}\text { Adoles- } \\
\text { cência }\end{array}$ & $\begin{array}{l}\text { - mudanças corporais produzem alterações na imagem corporal, sentimentos de vulnerabilidade do } \\
\text { corpo, sensação de difusão das fronteiras do corpo, } \\
\text { - apesar de parecer rápido o desenvolvimento da imagem corporal, modos mais complexos de } \\
\text { percepção corporal continuam a ocorrer até os 16,17 anos. }\end{array}$ \\
\hline
\end{tabular}

Para Fisher (1986), nessa sequência de desenvolvimento há três pontos de maior importância. 0 primeiro refere-se aos últimos meses do segundo ano de idade da criança, quando ela aprende a identificar seu espaço corporal como a única referência do seu eu $\mathrm{O}$ segundo ocorre entre os quatro e cinco anos, quando há um avanço marcante no domínio da sua percepção corporal, uma notável expansão do conhecimento do corpo e da habilidade de produzir representações desse corpo que são bem similares à realidade. 0 esquema corporal torna-se capaz de prover uma estrutura de defesa adequada para o enfrentamento de situações complexas ou "distorcidas". O terceiro ponto importante aparece aos nove anos, quando novamente acontece um avanço no conhecimento do corpo, na habilidade em elaborar descrições reais desse corpo e em manipular conceitos espaciais ligados a coordenadas corporais, ou seja, tendo o corpo como referencial. A adolescência, apesar de ser uma fase em que ocorrem muitas mudanças corporais e, consequentemente, a imagem corporal continua a se desenvolver, pouco ficou demonstrado empiricamente, apenas que existe um aumento visível na preocupação do adolescente com a segurança do seu corpo. 
No desenvolvimento da percepção corporal, foram encontradas algumas diferenças de gênero. A garota apresenta maior segurança em relação a seu corpo do que o garoto, ao mesmo tempo em que possui uma autocrítica muito maior ao julgá-lo verbalmente. Desde criança, a garota demonstra maior insatisfação com a aparência do seu corpo. Isso pode refletir o fato de que ela se sente menos defensiva que os garotos em relação a seu corpo ou, ainda, o fato de que, culturalmente, cobra-se que a mulher possua uma atratividade corporal. Para Fisher, a garota atinge um grau de segurança corporal antes do garoto, possui menos fantasias de destruição de seu corpo e lida melhor com as mudanças durante o desenvolvimento de sua imagem corporal (contrariando as suposições freudianas de que a garota sofreria de uma inferioridade corporal causada pela ausência do pênis). Segundo Fisher, a superioridade feminina de lidar com o próprio corpo e suas transformações resulta da sua necessidade de se adequar a um processo mais rápido de maturação corporal na adolescência (alterações corporais na puberdade, menstruação). De acordo com o autor, os garotos são, desde cedo, expostos a condições de perigo corporal, de agressividade e enfrentamento com 0 "inimigo", situações estas que podem lhes causar sentimento de destruição corporal, enquanto às garotas é passada a mensagem do potencial que seu corpo tem para gerar vidas e da necessidade de protegêlo.

Fisher (1986) analisou formulações teóricas e dados empíricos sobre o desenvolvimento da imagem corporal, fornecidos por inúmeros autores, como aqueles já citados anteriormente; realizou o cruzamento desses conhecimentos, indicando os aspectos que puderam e não puderam ser comprovados empiricamente; sumarizou as principais tendências sobre o desenvolvimento das percepções corporais que emergiram do material analisado e propôs que:

1. A criança vai tornando-se cada vez mais conhecedora do seu território corporal. Ela aprende muito sobre as dimensões espaciais do seu corpo e as funções dos órgãos e dos subsistemas corporais. 0 mapa do seu corpo vai, gradualmente, ficando mais complexo e diferenciado.

2. A criança é capaz de melhorar cada vez mais a habilidade de avaliar as propriedades físicas do seu próprio corpo.

3. O domínio precoce de certos aspectos da percepção corporal parece contribuir para um posterior domínio de habilidades cognitivas.

4. Aos poucos, a criança torna-se cada vez mais consciente das regras culturais e de padrões que definem alguns parâmetros para a atratividade física e para a classificação sexual.
5. Progressivamente, a criança intensifica 0 investimento em desenvolver a habilidade de segregar seu corpo do campo geral das percepções e de experienciá-lo como um objeto que possui um nível, mesmo que mínimo, de estabilidade. Existe uma preocupação com possíveis "invasões" ao corpo que podem violar essa separação ou constância. Há evidências de que as atitudes e comportamentos dos pais são fatores importantes na determinação do caráter da diferenciação da imagem corporal e na articulação das fronteiras corporais.

6. 0 processo de diferenciação sexual na imagem corporal não ocorre apenas a partir da constatação das diferenças estruturais do corpo feminino e masculino, mas também está relacionado a diferenças existentes entre os papéis sociais de cada gênero. As experiências de socialização femininas e masculinas provavelmente afetam aspectos da imagem corporal como, por exemplo, o sentimento de uma fronteira corporal segura.

7. Aparentemente, todas as fases de desenvolvimento da imagem corporal geram tensões. Por exemplo, a criança preocupa-se se seu corpo está dentro dos padrões de atratividade ou se os perigos externos podem romper suas fronteiras, desestabilizando sua integridade corporal.

8. Apesar de haver idades caracterizadas pela aceleração do desenvolvimento da imagem corporal, existem fases críticas para esse desenvolvimento. Geralmente, o controle do espaço corporal parece progredir gradualmente, e esse processo provavelmente se prolonga até o final da adolescência.

Os autores destacados por Fisher (Erikson, 1950; Freud, 1908; Piaget, 1954; Schilder, 1999; Werner, 1957) possuem um ponto de partida em comum. Para eles, a experiência corporal é responsável pelo desenvolvimento humano. A experiência corporal emerge, inicialmente, das primeiras percepções corporais da criança, das suas experiências sensóriomotoras. Tais experiências vão sendo internalizadas gradualmente, gerando imagens, ou seja, representações mentais do próprio corpo. Este fenômeno é que vai garantir o desenvolvimento cognitivo, a aquisição da linguagem e de outras habilidades.

Para Fisher, essas representações mentais das percepções corporais vão fazer com que, aos poucos, a criança se perceba como uma entidade única e separada do mundo que a cerca. Tal fenômeno, denominado processo de diferenciação, vai dar-lhe a noção de unidade. $\mathrm{O}$ que a separa do mundo externo é sua superfície corporal. O conceito de fronteiras corporais, proposto por Fisher e Cleveland (1968), representa essa superfície e aquilo que o indivíduo permite ou não 
permear sua imagem corporal. As fronteiras corporais delimitam distâncias e proximidades e oferecem a noção de um corpo seguro, protegido e menos vulnerável.

As primeiras percepções corporais vão influenciar 0 modo pelo qual o indivíduo interpreta o mundo e interage com ele, e as diferentes maneiras de reagir a determinado estímulo sempre estarão fundamentadas no princípio da manutenção de um corpo seguro. Os padrões de percepção corporal servem para específicos propósitos de defesa, pois vão informar se determinado estímulo deve ser evitado ou não. A reação corporal desencadeada por esse estímulo se dá em função da regulação da ansiedade. $\mathrm{E}$ a ansiedade é causada pela sensação de ameaça da integridade corporal.

\section{Considerações finais}

As contribuições de Fisher para a compreensão do desenvolvimento psicomotor estão vinculadas à sua abordagem psicodinâmica da imagem corporal, que considera os mecanismos psíquicos de defesa e proteção em prol de um corpo seguro e a complexidade individual de cada ser humano.

Os dados oferecidos por Fisher permitem-nos concluir sobre 0 importante papel da percepção corporal na construção da imagem corporal. A percepção corporal parece ser a primeira forma de experiência corporal vivenciada por todo indivíduo. É através das percepções que a criança vai se comunicando com o mundo e percebendo-se diferente dele. Ao aprimorar seus conhecimentos sobre 0 seu próprio corpo, vai fazendo deste o seu referencial para a compreensão e interpretação do mundo que o cerca. E a partir do corpo que ela começa a desenvolver outras habilidades fundamentais para a sua vida, como a cognição, a coordenação motora e a linguagem.

As experiências sensório-motoras da criança são favorecidas pelas suas primeiras relações objetais, estabelecidas, na maioria das vezes, com os pais, que lhe oferecem afeto e consistência para que ela discrimine uma percepção de outra. Nesse processo, aprende não só a diferenciar suas percepções, mas a se diferenciar do mundo, a perceber os diferentes estímulos advindos desse mundo e as diferentes reações corporais por eles provocadas. 0 processo de socialização da criança, que faz parte do seu desenvolvimento, tem como base o seu corpo e, apesar de haver indícios de que ao longo da vida ela tem como referência os fatores cognitivos para as tomadas de decisão, o corpo continua sendo uma matriz de registros das experiências vividas e de dispositivos de respostas possíveis aos estímulos recebidos. A experiência corporal adquire, então, um significado psicológico que vai influenciar inevitavelmente 0 comportamento do indivíduo. Aqui fica em evidência o caráter dinâmico da imagem corporal: a estabilidade de um eixo central que garante a identidade do sujeito e a dinamicidade para transformações, que permite a esse sujeito ressignificar suas experiências.

A singularidade de cada pessoa que dialoga com 0 tempo e o espaço e com tudo o que ocorre nesse tempo e espaço faz do ser humano um ser existencial. Compreender cada ser humano em sua complexidade e singularidade faz com que o profissional torne-se mais próximo da pessoa com quem está lidando, podendo, então, compartilhar experiências e auxiliar 0 seu processo de desenvolvimento.

A experiência do corpo é base para 0 desenvolvimento humano, e os conhecimentos produzidos por Fisher e aqui disponibilizados permitem aos profissionais que atuam na educação e na formação de crianças considerarem o corpo como 0 mediador das relações interpessoais estabelecidas em ambientes educativos. As habilidades motoras que vão sendo adquiridas pela criança em ambiente escolar, por exemplo, proporcionam o desenvolvimento do seu conceito de corpo e ampliam as possibilidades de novas experiências com 0 corpo em termos de percepções e movimentos, o que lhe oferece segurança para optar por caminhos alternativos em diferentes situações de sua vida.

O trabalho de Fisher também propõe que profissionais da saúde atuem sob o viés da imagem corporal, que irá lhes permitir ter uma abordagem mais complexa e individualizada, uma vez que esta considera a interação de aspectos fisiológicos, psicológicos e sociais no tratamento dos pacientes.

\section{Referências}

Cash, T. F. (2004). Body image: past, present and future. BootyImage 1(1), 1-5.

Cash, T., \& Pruzinsky, T. (Ed.). (2002). Body image a handbook of theery, rench and dinical practice Nova Iorque: Guilford Press.

Cervo, A. L., \& Bervian, P. A. (1975). Meoddoga dentífica para uso dos estudantes São Paulo: McGrawHill.

Erikson, E. H. (1950). Childhood and soidy. Nova Iorque: Norton.

Fisher, S. (1970). Body experience in fantasy and behavior. Nova Iorque: Appleton-Century-Crofts.

Fisher, S. (1986). Deudqumet and struture of the booly image Nova Jersey: LEA. 
Fisher, S. (1990). The evolution of psychological concepts about the body. Em T. Cash \& T. Pruzinsky (O rgs.), Booly images dadqpment, deiance and dange (pp. 3-20). Nova Iorque: The Guilford Press.

Fisher, S., \& Cleveland, S. (1968). Booty image and pessonality. ( $2^{\mathrm{a}}$ ed.). Nova Iorque: Dover Publications.

Freud, S. (1908). Character and anal erotism. Em J. Strachey (Ed.). The standard exition of the complete psychdogical works of Sigmmd Fread (pp. 169-175). Londres: Hogarth. (O riginal publicado em 1959).

Gellert, E. (1960). A deudqumetal study of dildren's idkess about the content and funtion of the human booty. Trabalho apresentado no Annual Meeting of the Eastern Psychological Association.

Klein, M. (1932). Thepsychoanalysis of dildren Londres: Hogarth.

Kohlberg, L. (1966). A cognitive developmental analysis of children's sex-role concepts and attitudes. Em E. E. Maccoby (Ed.), Thedeudqpment of sex differenes (pp. 82-173). Stanford: Stanford University Press.

Lakatos, E. A., \& Macconi, M. A. (1991). Fundamentos demtoddoga ȧentífica. São Paulo: Atlas.
Piaget, J. (1954). The construction of reality in the dildren Nova Iorque: Basic Books.

Piaget, J. (1960). The dild's conception of the word Paterson, NJ: Littlefield, Adams.

Reich, J. (1949). Charadter analysis Nova Iorque: O rgone Institute.

Salvador, A. D. (1982). Méodos e ténicas de pesquisa bibliogáfica: dabaração de trabalhos detíficos Porto Alegre: Sulina.

Schilder, P. (1999). A imagem do copa as enegias construtivasda psique São Paulo: Martins Fontes.

Werner, H. (1957). Comparative psydhdogy of mental dadopmant Nova Iorque: International Universities Press.

Williams, P. D. (1979). Children's concepts of illness and internal body parts. Matemal-Child Nursing Jaumal, 8, 115-123.

Witkin, H. A., Dyk, R. B., Faterson, H. F., Goodenough, D. R., \& Karp, S. A. (1962). Psychological differentiation. Nova Iorque: Wiley.

Reedbidbem10/ 08/ 2010

Reformuladbem20/ 08/ 2012

Aprovadb em08/ 09/ 2012 
Sobre as autoras:

Patrícia Russo Leite Ribeiro é graduada em Psicologia pela Universidade Federal de São Carlos (UFSCar), especialista em Atividade Motora Adaptada e mestre em Atividade Física Adaptada pela Faculdade de Educação Física da Universidade Estadual de Campinas (UNICAMP). Arte Educadora na Coordenadoria de Artes e Cultura da Prefeitura Municipal de São Carlos (PMSC), desenvolveu projetos sociais junto à Secretaria Municipal de Cidadania e Assistência Social e à Secretaria Municipal Especial da Infância e Juventude (PMSC).

Maria da Consolação Gomes Cunha Femandes Tavares é graduada em Medicina e Fisioterapia, mestre e doutora em Medicina Interna pela FCM/ UNICAMP. Possui formação em Psicanálise pela CPCamp. Livre-docente em Imagem Corporal e professora titular do DEAFA (Departamento de Atividade Física Adaptada), na FEF/UNICAMP, autora de três livros sobre imagem corporal e de vários artigos científicos nacionais e internacionais, e líder do GEPIC - Grupo de Estudos e Pesquisa em Imagem Corporal, cadastrado desde 2001 na Plataforma Lattes.

Aletha Silva Caetano é bacharel e licenciada em Educação Física pela Universidade Estadual de Campinas UNICAMP, especialista em Atividade Motora Adaptada - UNICAMP, mestre e doutora em Atividade Física, Adaptação e Saúde pela Faculdade de Educação Física - UNICAMP. Professora da Universidade Nove de Julho UNINOVE, atua na linha de pesquisa em Saúde da Mulher.

Contato com as autoras:

Rua Joaquim Gomes Pinto, 43, apto. 14 - CEP: 13025-010. Cambuí - Campinas-SP.

E-mail: pirusso@uol.com.br 